\title{
Readability: The Contextual Hindrance for Teenage Readers
}

\author{
Hadjeres Sarra \\ Canterbury Christ Church University
}

\begin{abstract}
Coursebook has always been one of the focal elements in foreign language teaching and learning research. Listening, speaking, reading and writing are the main skills that construct this medium. Reading seems to be one of the challenging tasks for foreign language learners especially in their first stages of learning the language. Reading in the foreign language may not be established through only knowing the meaning of the most frequently used words or through deciphering the collocating relationship between them. It seems to be a systematically sophisticated process that can be mysteriously achieved through working on a set of factors. This paper looks at the readability level of the English textbooks used in the Algerian middle schools. There is a noticeably large number of Algerian learners with reading problems. This study is significant in that it tends to look at reading from a contextual perspective as well as it seeks to uncover the social, cultural, personal factors that may either impede or foster teenage readers' text comprehension and readability. Readability in this paper is discussed from a qualitative perspective since previous research on this were more concerned with looking at text difficulty and appropriateness through using a number of quantitative readability formulas and measurements. The study aims at figuring out how can 'outside text' indices affect text-reader interaction and understanding. This work tends to gain a good understanding of the relationship between the text and the reader and see how non-text factors either foster or hinder the comprehension process. The study is conducted following a qualitative approach since the researcher is more concerned with readers' and teachers' views, experiences and prejudices about reading texts included in the school books. Data are collected through implementing individual interviews with teachers and coursebook developers, focus group interviews and group discussions with middle school learners. The findings reveal that the readability of text is not only textual, however; it is governed by other contextual features that intervene in the reading comprehension process.
\end{abstract}

Keywords: Comprehension; Culture; Middle School; Schemata; Social backgrounds. 